\title{
Numerical modelling of braiding and meandering instabilities in
}

\section{gravity-driven liquid rivulets*}

\author{
Daniel Sebastia-Saez ${ }^{1}$, Tomas Ramirez Reina ${ }^{1}$, Harvey Arellano-Garcia ${ }^{1 \bowtie}$ \\ *Dedicated to Prof. Dr.-Ing. Günter Wozny on the occasion of his 70th birthday \\ ${ }^{\triangle}$ Corresponding Author:Prof. Dr.-Ing. Harvey Arellano-Garcia (h.arellano-garcia@surrey.ac.uk)
}

\begin{abstract}
Rivulet instabilities appear in many engineering applications. In absorption equipment, they affect the interface area available for mass transfer, and thus, reducing the efficiency. We use Computational Fluid Dynamics to reproduce the meanders and braids in rivulets flowing down an inclined channel. Fast oscillations of the meander $(\mathrm{f}=5.6 \mathrm{~Hz})$ are observed at low flow rates. At greater flow rates, an analysis of the transversal velocity in the retraction waves shows the effect of the surface tension, which causes the braiding phenomenon, and thus, the reduction in gas-liquid interface area.
\end{abstract}

Keywords: rivulet flow; Navier-Stokes equations; meandering; braiding; Computational Fluid Dynamics; multiphase modelling

\section{Introduction}

The hydrodynamics of rivulets has been an open problem in recent decades, with applications in absorption, heat exchange, microfluidics, and distillation [1]. The flow of a liquid phase running down a solid substrate has been studied in the past using both experimental and computational methods - usually with the purpose of optimizing the gas-liquid interface by

${ }^{1}$ Dr. Daniel Sebastia-Saez, Dr. Tomas Ramirez Reina, Prof. Dr.-Ing. Prof. h.c. Harvey ArellanoGarcia.

Department of Chemical and Process Engineering, University of Surrey, GU2 7XH, United Kingdom. 
varying the physical properties of the liquid [2-10]. Less attention, however, has been directed towards the fundamentals of the instabilities inherent to such flows; namely, meandering and braiding. Deeper knowledge is, therefore, needed in this regard so as to control and optimize the multiphase flow in inclined channels, since for instance, mass transfer in absorption equipment is affected by those instabilities, which can result in a loss of gas-liquid interface area $[11,12]$. The hysteresis phenomenon in the relation between the interface area and the flow rate is another open question. This phenomenon was observed by Iso et al. [13], who reported that the solid area covered by the liquid phase depends on the previous liquid flow rate history. In particular, greater interface area appears for decreasing flow rates. Despite its implications on the total gas-liquid interface area available for mass transfer, the cause of this phenomenon was not profoundly discussed by the authors.

Meandering and braiding have been investigated from the experimental and theoretical perspective. Several mechanisms have been pointed out as the cause of meandering, including dynamic instabilities at the gas-liquid interface, upstream liquid flow rate fluctuations, and contact angle hysteresis. Daerr et al. [14] emphasised the role of the dynamic imbalances at the gas-liquid interface as the origin of this disturbance. The authors performed experiments upon complete wetting conditions to remove the effect of the solid surface microstructure. Their analysis is, therefore, based on the assessment of the terms in the two-dimensional NavierStokes equations. The authors found that the difference between the average velocity and the phase velocity of the rivulet determines whether or not meandering occurs. Other authors point out the substrate microstructure and contact angle hysteresis as the main source $[15,16]$. Birnir 
et al. [12] stated that meandering happens because of upstream disturbances in the liquid flow rate.

The mechanism of meandering is therefore an open issue, and thus, further research must be carried out to elucidate what is the cause of the formation of meanders in gravity-driven rivulets. As Daerr et al. [14], our intention is to suppress any influence of contact angle hysteresis and liquid flow fluctuations. This may be attained by means of numerical simulations. To the best of our knowledge, there are no CFD studies dealing with the analysis of the said instabilities in the literature. Accordingly, we have conducted a CFD study aiming to tackle the unresolved questions of this fundamental aspect of wetting.

As for braiding, Mertens et al. [11] established that in the absence of liquid flow rate perturbations, the liquid rivulet alternates between full film flow and braiding areas. A mathematical criterion (depending on the liquid viscosity, flow rate, surface tension, liquid density, and effective acceleration of gravity) was presented to predict the formation of braids. Also, the authors described the mechanism of the braid formation as a balance between the inertia and the surface tension. We have compared the results obtained in our CFD simulations with the aforementioned criterion, with the results showing fair agreement with it.

This work presents a CFD analysis of both meandering and braiding instabilities. The oscillation of the rivulet meanders and the formation of braids are described. Design directions are given as to how to control them.

\section{Numerical approach and grid independence check}

A computational domain consisting of a rectangular channel with a flat inclined plate is used to study the development of the liquid rivulets. Figure 1 shows a schematic illustration of the 
computational domain, which mimics a wall on which the liquid rivulet forms. The plot also shows the inclination angle $\alpha$ over the horizontal plane, the boundary conditions (including the contact angle $\theta$ in the walls and the liquid volume fraction $F$ in the liquid inlet) and the reference frame. A schematic of the structured mesh used is also depicted with the densification applied in the vicinity of the wall. The air-water system is used in all the simulations. The liquid inlet consists of a thin slot occupying the entire width of the computational domain and with a thickness $\delta$, which varies from 0.2 to $0.4 \mathrm{~mm}$. This range of values corresponds to the thickness of liquid films in industrial applications as indicated by Haroun et al. [17].

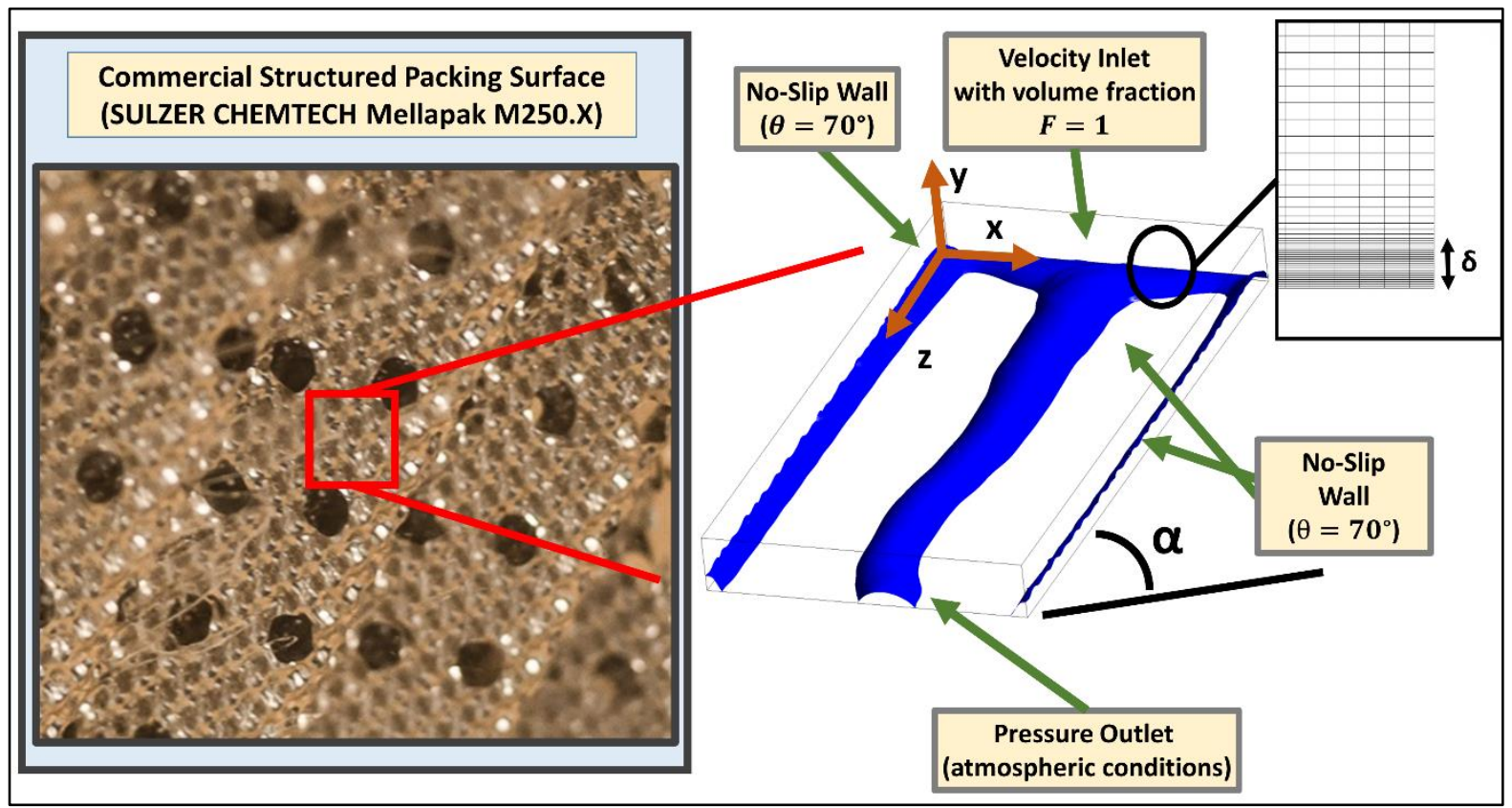

Figure 1 Schematic illustration showing the relation between the computational domain (on the righthand side) and a solid wall with the liquid rivulets. The image on the left-hand side corresponds to a commercial packing surface.

The pressure-based solver available in the package ANSYS Fluent v14.0 was used to run the calculations. The second order upwind scheme is selected for space discretisation. Transient simulations are run with a variable time step keeping the Courant number constant at 0.5 . The PRESTO! algorithm is used for pressure correction. The laminar option is chosen owing to the 
low Reynolds number considered $\left(\operatorname{Re}=\frac{\rho v_{i n l} \delta}{\mu}<171\right)$. The Volume of Fluid Method (VoF) along with the Geo-Reconstruct algorithm are used to track the gas-liquid interface. The VoF method solves the same set of conservation equations for all the phases involved. Upon those circumstances, the density $\rho$ and the dynamic viscosity $\mu$ are not constant throughout the domain, although incompressible fluids are used. Instead, both density and viscosity are averaged by the volume fraction of each phase in the computational cell. Taking the partial derivatives of the density $\rho$ with respect to time $t$, the mass conservation equation is rewritten as

$$
\frac{D \rho}{D t}+\rho(\nabla \cdot \vec{v})=S_{m}
$$

where $\vec{v}$ is the velocity vector and $S_{m}$ is the mass source term, which describes the reactive mass transfer from the gas into the liquid phase. Eqn. (1) can be combined with the momentum conservation equation to obtain the following single governing equation

$$
-\nabla p+\mu \nabla^{2} \vec{v}+\mu \nabla(\nabla \cdot \vec{v})+\vec{S}_{\mu}+\rho \vec{g}+\vec{S}_{\sigma}=\vec{v} S_{m}-\vec{v} \rho(\nabla \cdot \vec{v})+\rho \frac{D \vec{v}}{D t}
$$

$S_{\mu}$ groups the terms which account for the derivatives of the dynamic viscosity $\mu . \vec{g}$ is the acceleration of gravity, and $p$ is the pressure field. The right-hand side of the Eqn. (2) shows that the derivative of the linear momentum corresponds to the sum of a term accounting for the mass change of the system and two terms describing the velocity change. $\vec{S}_{\sigma}$ stands for the surface tension momentum source, which is implemented according to the Continuous Surface Force (CSF) model as

$$
\vec{S}_{\sigma}=\gamma_{l g} \frac{\rho \boldsymbol{\kappa} \nabla F}{\frac{1}{2}\left(\rho_{l}+\rho_{g}\right)},
$$

where $\boldsymbol{\kappa}$ represents the curvature of the gas-liquid interface, $\gamma_{l g}$ is the surface tension coefficient, $F$ indicates the volume fraction of the liquid phase, $\rho$ is the density, and the 
subscripts $l$ and $g$ denote liquid and gas phase, respectively [18]. No slip condition along with a constant value of the contact angle $\left(\theta=70^{\circ}\right)$ was used at the walls.

Moreover, a grid independence study was performed to find the optimum grid size. Two cases were tested: Case 1 and Case 3 (see Tab. 2). The dimensions of the plates are expressed all throughout this work as width of the domain $\times$ liquid inlet thickness $(\delta) \times$ length of the domain. The details of the three grids are shown in Tab. 1, with the grid spacing $h$ being in the range between 8.3 and $13.8 \mu \mathrm{m}$.

The Richardson extrapolation was applied to study the grid convergence and give an estimation of the grid discretization errors. The Richardson extrapolation states that the numerical solution $\Phi$ of a given problem equals the exact solution $\Phi_{\text {exact }}$ of the equations plus a series of correction terms as

$$
\Phi=\Phi_{\text {exact }}+g_{a} h+g_{b} h^{2}+H \cdot O \cdot T
$$

where H.O.T. stands for higher order terms and $g_{a}$ and $g_{b}$ are continuous functions which do not depend on the grid spacing $h$. With two different grids, dropping the higher order terms and considering that a second order method is used to solve the equations, the following expression is obtained

$$
\Phi_{\text {exact }} \cong \Phi_{1}+\frac{\Phi_{1}-\Phi_{2}}{r^{\tilde{p}}-1}
$$

which establishes that the exact solution can be expressed as the solution with the fine grid $\phi_{1}$ plus a correction term that depends on the grid refinement ratio $r=N_{1} / N_{2}=N_{2} / N_{3}$ (being $N$ 
the number of nodes of the grids), the apparent convergence order $\tilde{p}$, and the difference between the value obtained with the fine grid $\Phi_{1}$ and that obtained with the medium grid $\Phi_{2}$.

From the correction term introduced in Eqn. (5), one can define the grid convergence index GCI as

$$
G C I_{12}=F_{S} \frac{|\varepsilon|}{r^{\tilde{p}}-1}
$$

where $F_{S}$ is a safety factor, which is equal to 1.25 , when three grid levels are used in the study, and equal to 3, when two grid levels are used [19]. The relative error $\varepsilon$ is defined as

$$
\varepsilon=\frac{\Phi_{1}-\Phi_{2}}{\Phi_{1}}
$$

To apply the Richardson extrapolation, the results need to fall within the asymptotic range of convergence. This condition is expressed as

$$
\frac{G C I_{23}}{r^{p} G C I_{12}} \approx 1
$$

Fig. 2 shows the results obtained for the grid convergence study of the interface area for both cases tested (Case 1 and Case 3). The interface area is normalized by the area of the solid surface, i.e. only the bottom surface of the plate, that is, excluding both lateral walls. The gasliquid interface is obtained as the isosurface with the volume fraction equal to 0.5 in the entire domain. In case 1 , the grid convergence indexes are $G C I_{12}=1.42 \%$ and $G C I_{23}=2.53 \%$, respectively, with an apparent order of convergence $\tilde{p}=4.85$. The condition in Eqn. (8) was evaluated and the results are satisfactorily within the asymptotic range $\frac{G C I_{23}}{r^{p} G C I_{12}} \approx 1.0088$. 


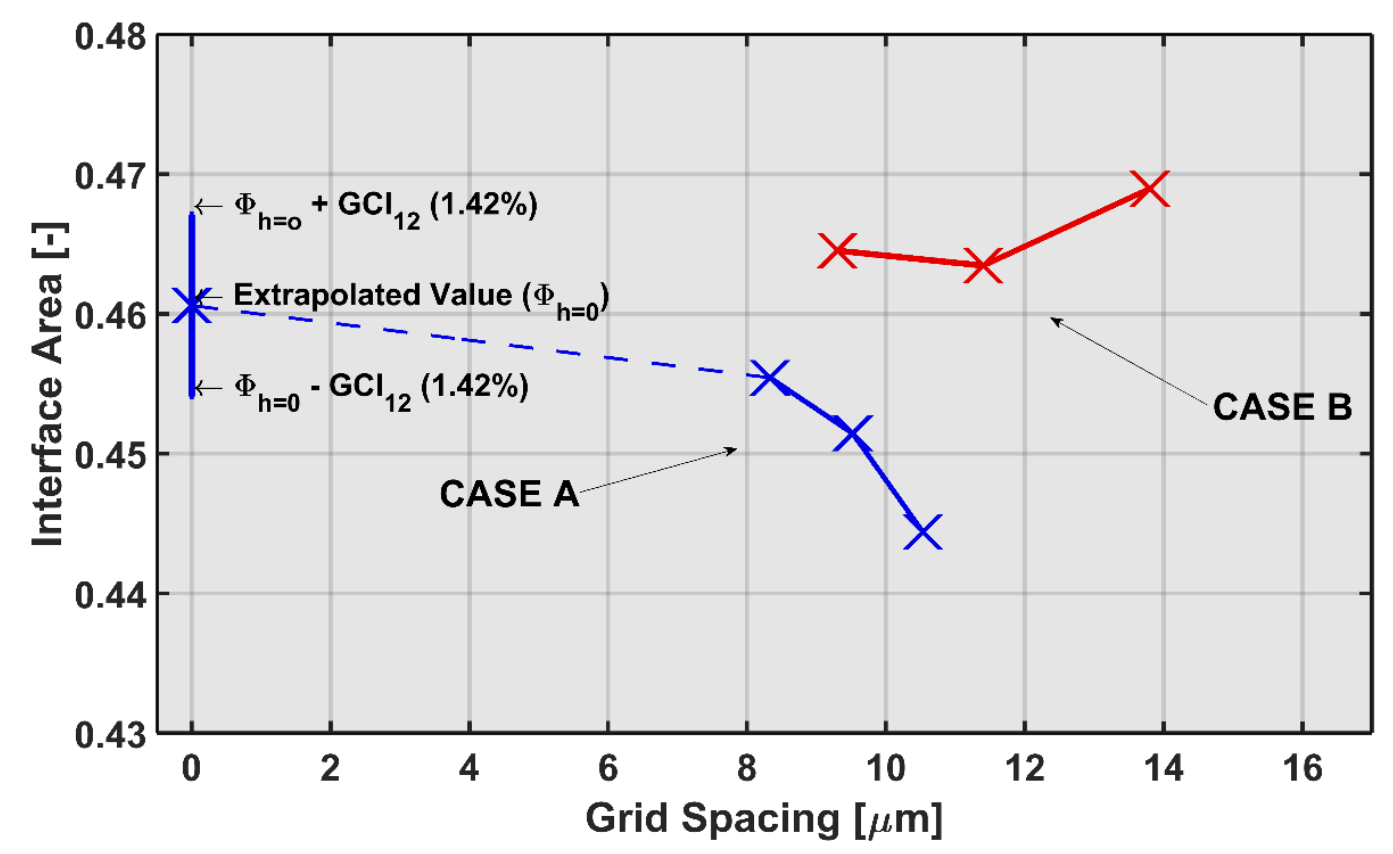

Figure 2 Grid convergence of the gas-liquid interface area. The continuous line highlights the tendency. The dashed section continues towards the extrapolated value at zero grid spacing $\left(\phi_{h=0}\right)$.

The final solution in that case is, therefore, the extrapolated value at zero grid spacing $\Phi_{h=0}$ with the error bar defined by the grid convergence index $\left(G C I_{12}\right)$.

Case 3, however, presents an oscillatory behaviour. In those instances, the final value is given by the average value, whereas the error is defined as half the difference between the maximum and minimum value observed [20].

A small difference between the results obtained with the three grids is observed for both cases, which allows to conclude that the grid spacing used in this study is adequate. The grid spacing of the simulations in this article are all within the range tested $(8.3-13.8 \mu \mathrm{m})$.

\section{Results and discussion}

Mertens et al. [1] obtained a correlation between two dimensionless quantities $\Pi_{1}$ and $\Pi_{2}$, which depend on the physical parameters of the liquid (density $\rho$, kinematic viscosity $v$, and surface tension $\gamma$ ) as well as on the flow rate $q$, and the plate inclination $\alpha$. The correlation 
allows to establish the boundary between braiding and non-braiding conditions. The results of six different test cases have been compared to the aforementioned correlation in order to give more confidence in the obtained results (see Figure 3). The conditions of all test cases are summarised in Tab. 2, including also the size of the domain in each case, which has been modified to accomodate the small flow rate of the non-braiding cases to the rivulet flow. Had the small liquid flow rate of cases 1 and $2\left(0.75 \mathrm{~cm}^{3} / \mathrm{s}\right)$ been implemented in the domain used for cases 4,5 , and $6(5 \times 0.04 \times 6)$, the Reynolds number $\left(\operatorname{Re}=\frac{\rho v_{i n l} \delta}{\mu}=16.85\right)$ would correspond to trickling flow, and thus impeding the visualisation of the rivulet. The six cases studied in this work have Reynolds numbers between 56.17 and 170.79, which correspond to rivulet flow according to the classification of Hoffmann et al. [10].

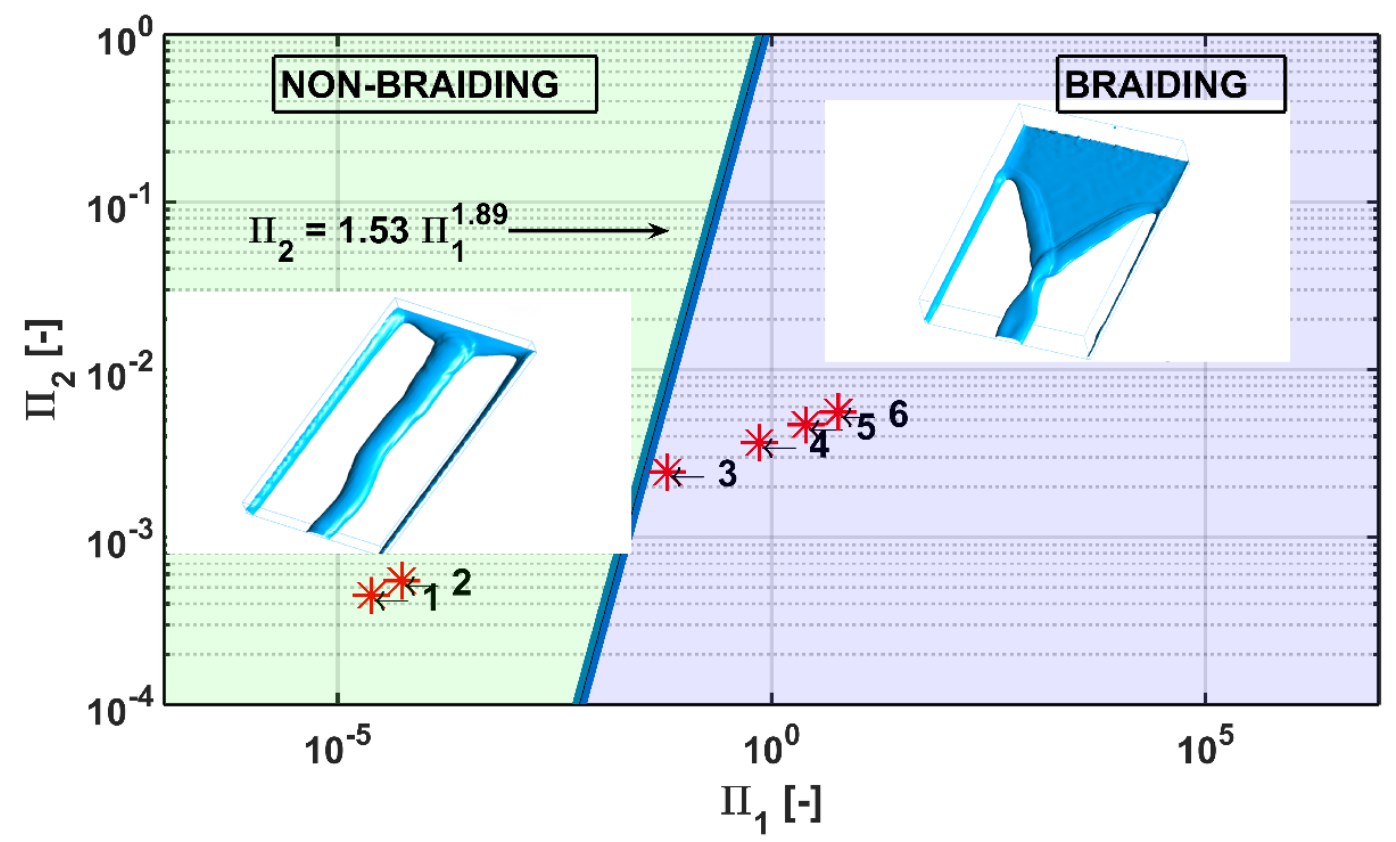

Figure 3 Braiding and non-braiding zones as a function of the physical properties of the liquid, the plate inclination, and the flow rate. The correlation given by Mertens et al. [1] delimits both zones with $\Pi_{1}=1 / 2 v \rho^{7} q^{5}(g \sin \alpha)^{4} \gamma^{-7}$ and $\Pi_{2}=1 / 2 v \rho^{2} q(g \sin \alpha) \gamma^{-2}$, respectively. 

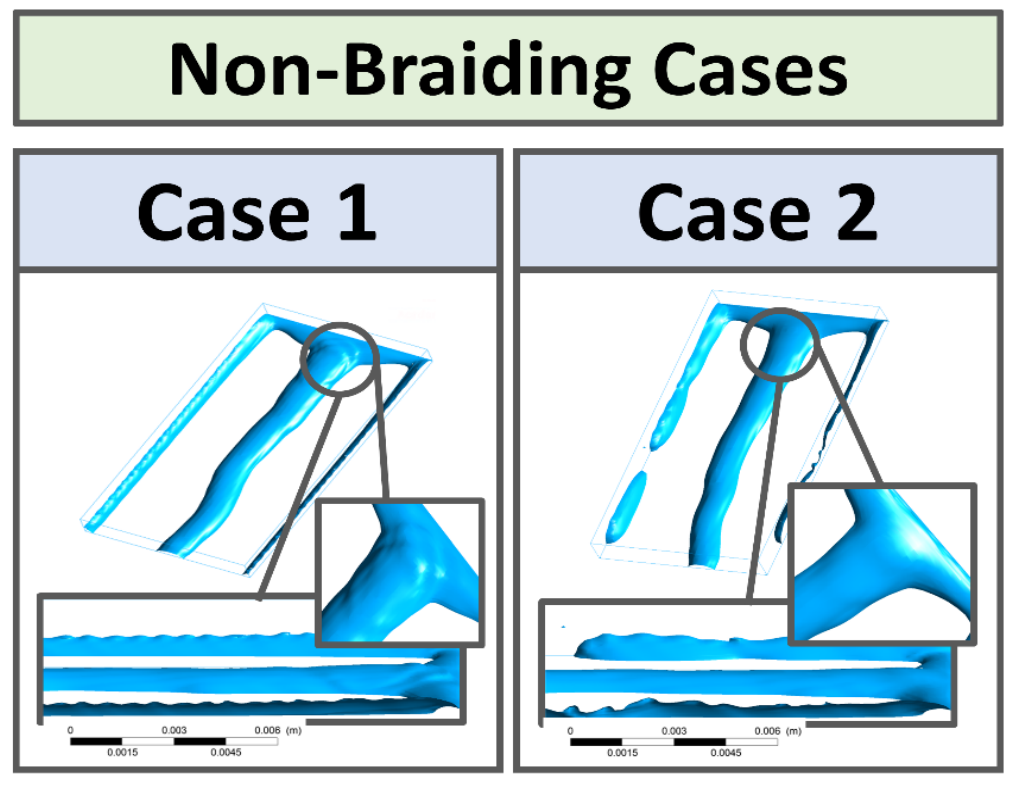

\section{Transition Case}

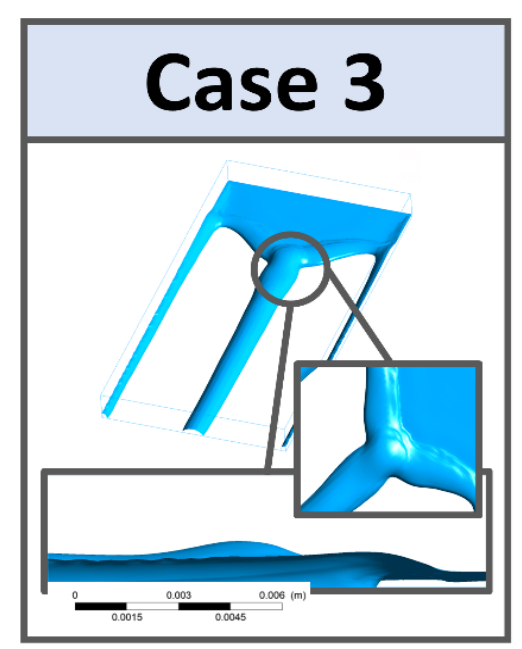

\section{Braiding Cases}
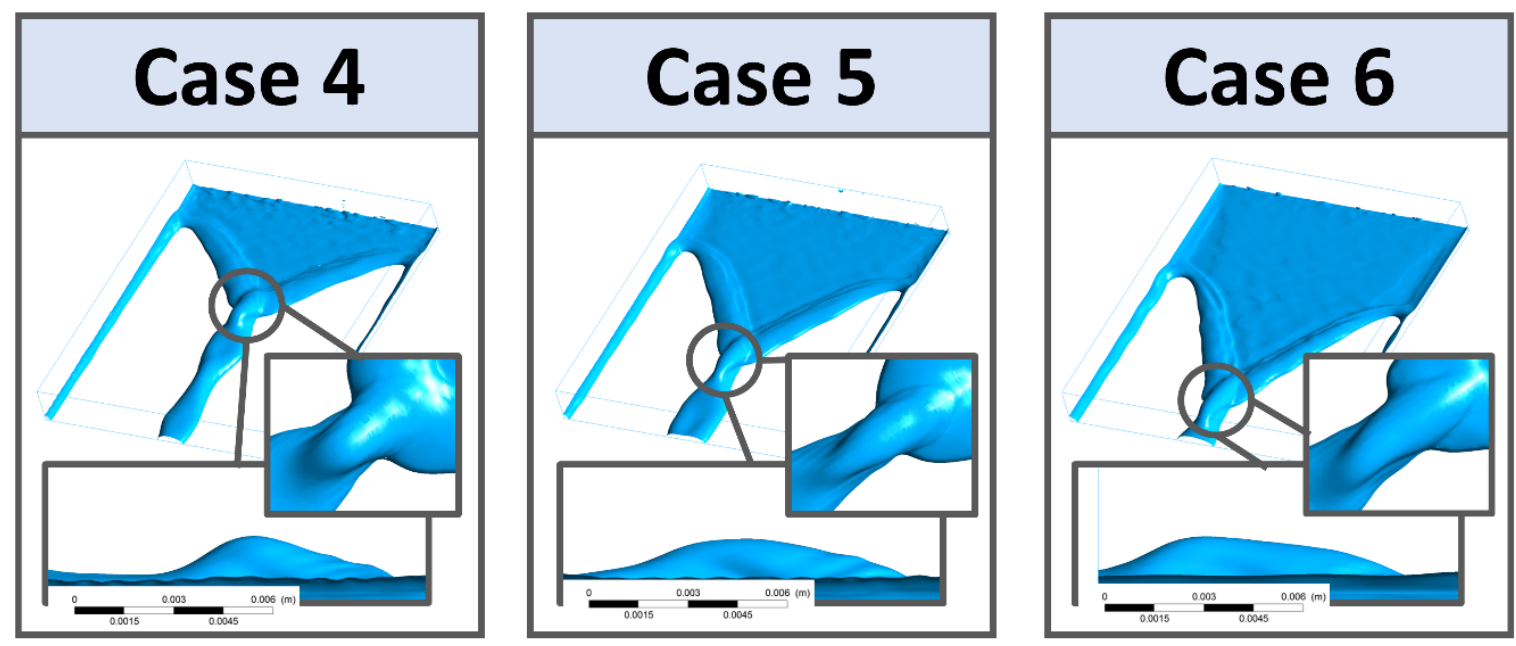

Figure 4 Comparison of the morphology of the six cases presented in Tab. 2. The plot includes top and side views in order to clarify the morphology of the braid or its absence.

Fig. 4 presents a comparison between the morphologies of the six cases presented in Tab. 2 and helps to visualise the braid, or its absence, in all of the cases considered. An additional 
case (case 3) is included with conditions corresponding to the transition between nonbraiding and braiding. In case 3 , there is an incipient braid, but its height is not as pronounced as in cases 4,5 , and 6 . In cases 4,5 , and 6 , one can notice that the formation of the braid occurs further from the liquid inlet as the liquid flow rate increases, since the extension of the fully developed film increases with the flow rate.

Meandering has been observed in Case 1 . In this case, the small volumetric flow rate $(0.75$ $\mathrm{cm}^{3} / \mathrm{s}$ ) does not allow the formation of an area with a fully developed liquid film, but the Reynolds number $\left(R e=\frac{\rho v_{i n l} \delta}{\mu}=56.18\right)$ is enough to form a rivulet (Hoffmann et al. [10]). Figure 5A shows the transient behaviour of the distance between the centre line of the computational domain and the symmetry axis of the rivulet at the outlet. The flow time $\tau$ is normalized by the characteristic residence time, i.e. length of the plate divided by the inlet velocity. The data series starts at $\tau=1.42 \mathrm{~s}$, when the rivulet first reaches the outlet. A stable period begins with no oscillations, which lasts until approximately $\tau=3.08 \mathrm{~s}$. After that, the meandering phenomenon is triggered and presents a frequency of $f=5.6 \mathrm{~Hz}$. The maximum deviation is approximately $1.5 \mathrm{~mm}$ towards either side of the centre line. Figure 5B depicts the position of the rivulet cross-section at the outlet for the semi-period indicated in the subplot A, whereas Figure 5C gives the vertical view, in which the oscillating behaviour of the entire rivulet is observed. A constant velocity at the inlet was set $\left(v_{i n l}=25 \mathrm{~cm} / \mathrm{s}\right)$, with no upstream flow rate fluctuations. Also, the plate is a smooth surface with a constant contact angle $\theta=70^{\circ}$, which removes any effect of contact angle hysteresis. Upon these circumstances, the 
meandering must appear owing to the evaluation of the terms in the Eqn. 3, as suggested by Daerr et al. [14].

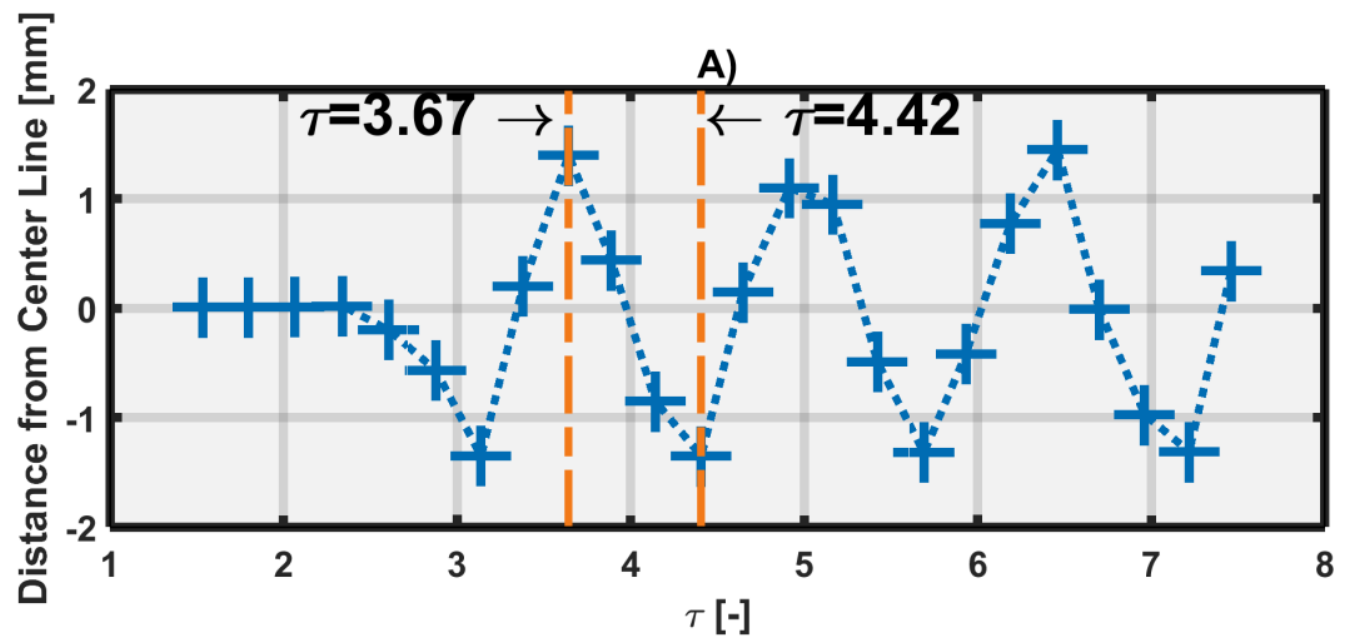

B)

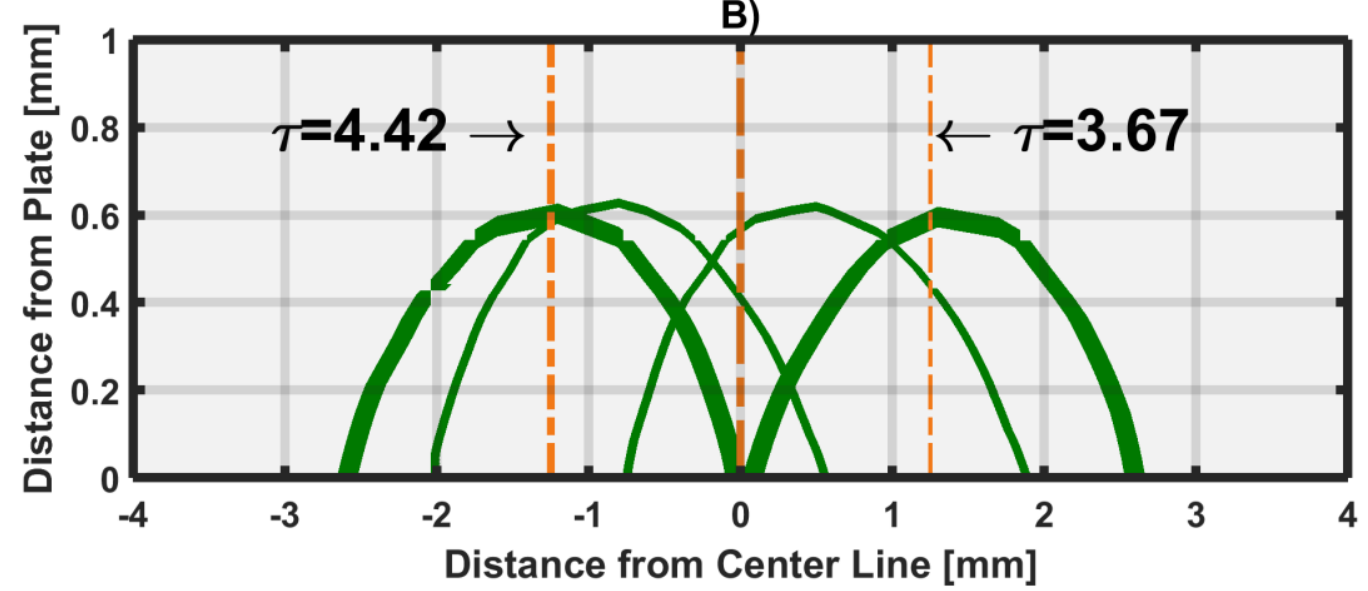

C)

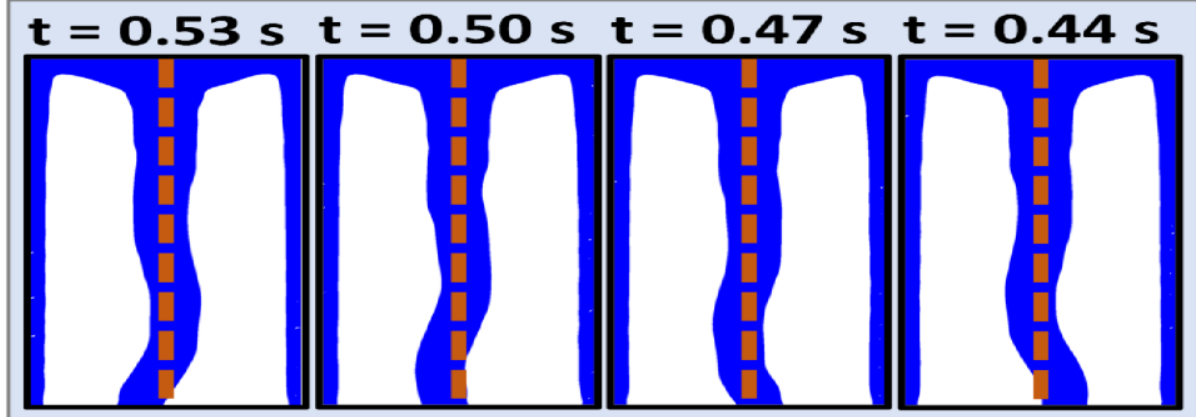

Figure 5 Schematic illustration of the meandering phenomenon in case 1: Transient position of the rivulet with respect to the centre line of the domain for the semi-period $\tau \in[3.67 ; 4.42]$. 
A deeper analysis of the braiding cases can elucidate the effect of surface tension, which hinders the liquid spreading. Fig. 6 shows the rivulet profile at the centre line of the domain and the total velocity isolines for case 5 . The velocity profiles are normalised by the velocity at the liquid inlet $(32 \mathrm{~cm} / \mathrm{s})$. The distance from the wall and the distance from the outlet are normalised by the dimensions of the computational domain.

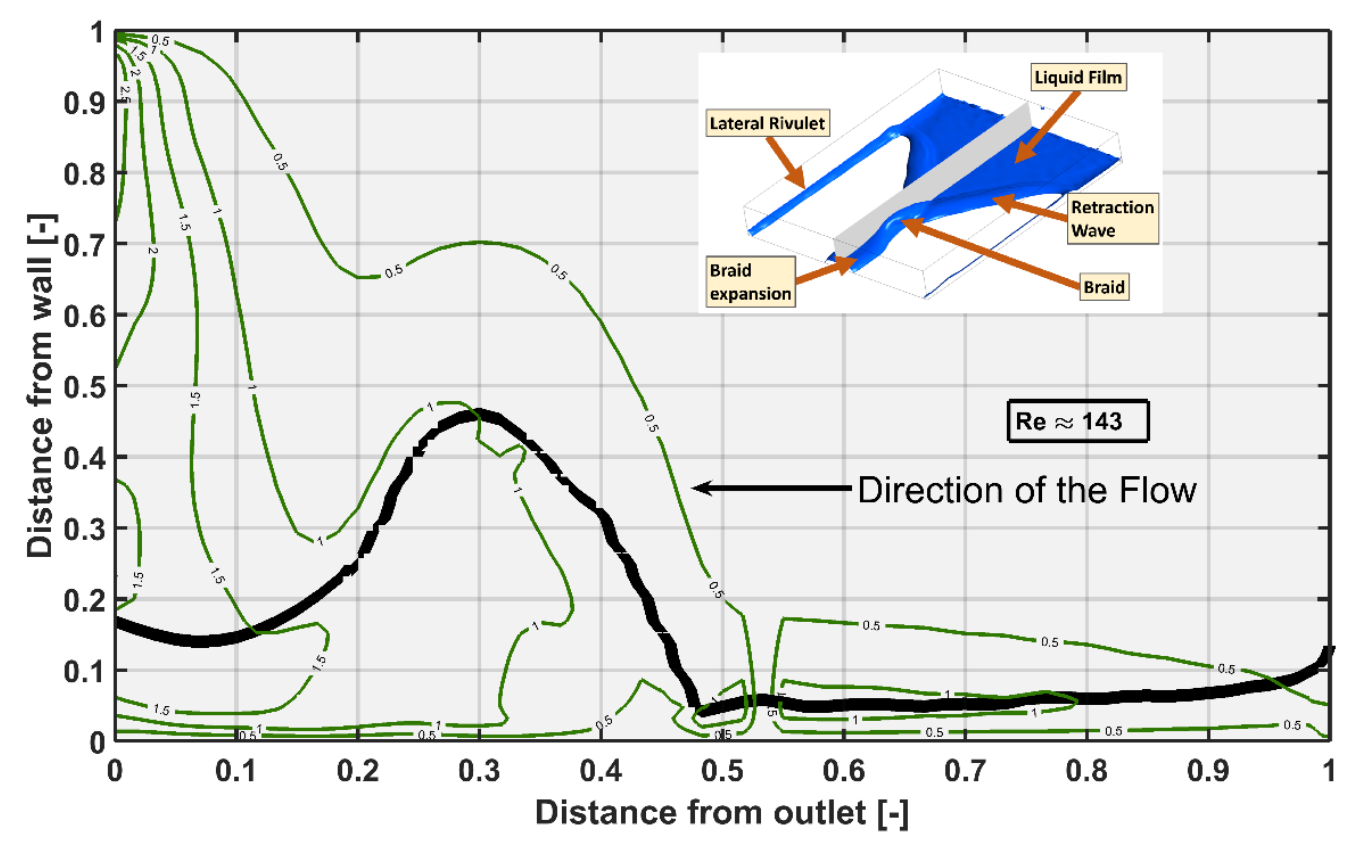

Figure 6 Total velocity isolines in the central profile of the rivulet flow for case 5 . The inset plot shows the liquid pattern. The observation plane is marked in grey. The thick black line denotes the gas-liquid interface.

Mertens et al. [1] explained the formation of braids as a continuous readjustment between the inertia of the liquid and the surface tension. The liquid spreads as it enters the domain giving rise to an area where a fully developed liquid film appears (see inset in Fig. 6 for details on the different areas of the liquid pattern as well as the observation plane). The greater the inertia of the liquid at inlet conditions, the greater the extension of the fully developed liquid film, which can exceed a certain equilibrium between the inertia and the surface tension. When that occurs, the surface tension brings back the liquid to the centre line, generating the braid as both 
retraction waves bounce against each other, due to the increase of momentum in the transversal direction as the liquid flows downwards. After the formation of the braid, gravity spreads the liquid again (braid expansion) and eventually another fully developed liquid film might appear. The process continues until damped by viscous effects, giving rise to the formation of a clearly defined rivulet. Further work will consist in implementing the volumetric flow rate of cases 4, 5 , and 6 in a longer domain allowing the visualisation of the formation of the rivulet after the braid to study whether meandering occurs upon those conditions.

The pattern described by Mertens et al. [1] is reproduced in Fig. 6, where the gas-liquid interface (thick black line), as well as the total velocity isolines are depicted. The fully developed film appears evident (at a distance from the outlet between 0.5 and 1), with an increase in the velocity from 0.5 to 1 . After the braid (distance from the outlet between 0 and 0.2 ), gravity accelerates the liquid again, with the normalised velocity presenting the greatest values $\left(v_{\text {norm }}>1.5\right)$ so as to keep the volumetric flow rate constant, provided the rivulet has less cross-section than the liquid film. 


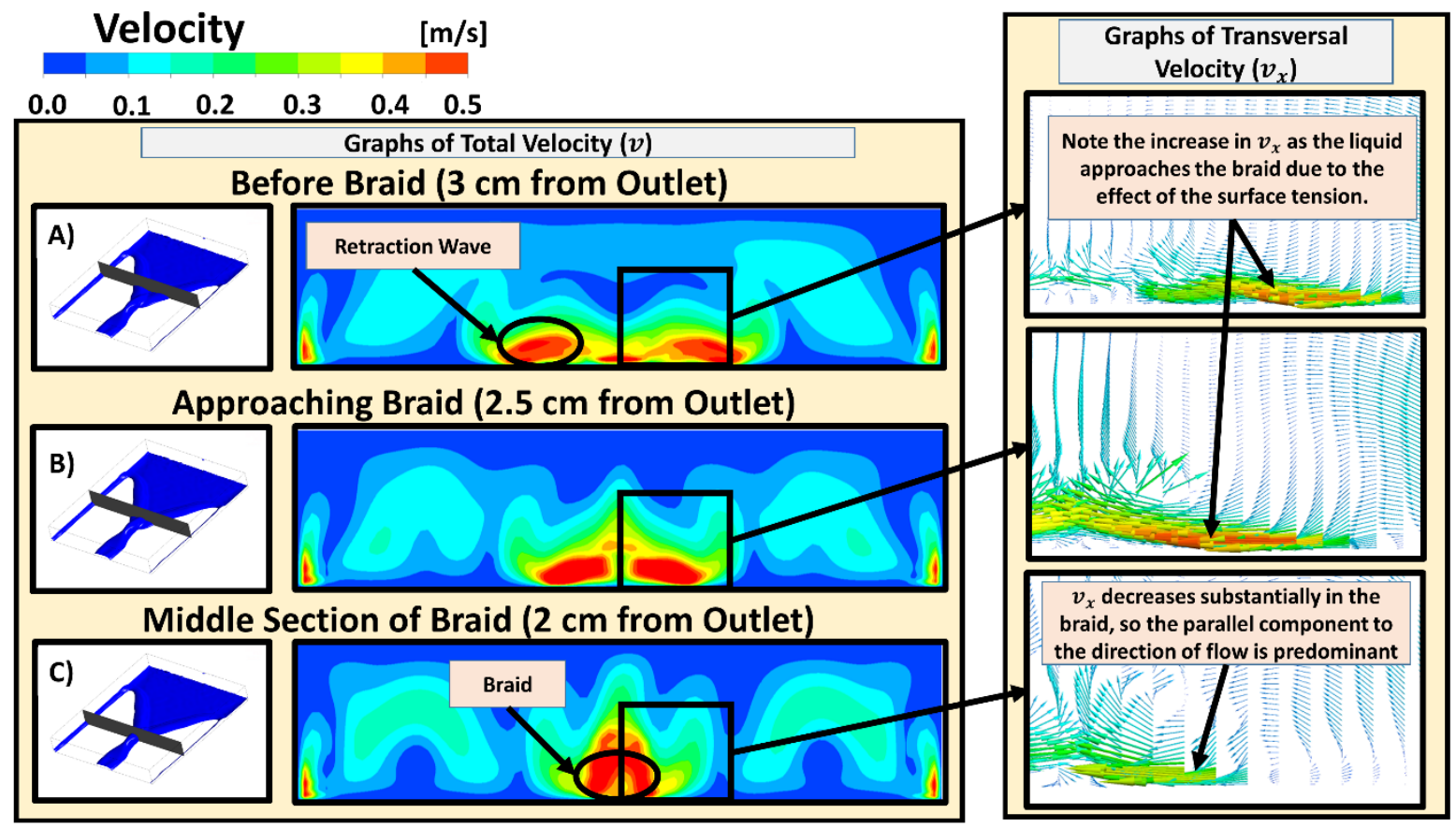

Figure 7 Total velocity contours (modulus of the velocity vector) at different cross-sections for case 5. Observation planes depicted in the subplots on the left-hand side. The subplots on the right-hand side show vector maps of the transversal velocity component only.

Fig. 7 illustrates the effect of the surface tension by analysing both the total and the transversal velocity of the liquid pattern, which have been taken at three successive parallel observation planes (see the subplots on the left-hand side of Fig. 7). The contour plot at $3 \mathrm{~cm}$ and $2.5 \mathrm{~cm}$ from the outlet are located before the braid (subplots A and B), whereas the plane at $2 \mathrm{~cm}$ from the outlet is placed at the middle section of the braid (subplot C). One can distinguish the parts of the cross-section occupied by the liquid phase, which have greater total velocity than the surrounding gas phase (depicted in red). The vector plots on the right-hand side show the transversal velocity in order to check the effect of the surface tension. One can see an increment in the transversal velocity as the liquid approaches the braid, which eventually causes both retraction waves to bounce, and thus, giving way to the braid. The latter is characterised by a relaxation of the transversal velocity component (see bottom transversal velocity graph), meaning that all the momentum of the liquid phase is directed down the plate. This process should repeat itself until damped, and it is possible to see in Fig. 4 (cases 4 and 5) that the liquid 
tends to form a second area with a fully developed liquid film and a subsequent second braid. It would be necessary, however, to use a bigger computational domain in order to see this effect clearly, and to quantify the damping effects that cause the appearance of the well formed rivulet predicted by Mertens [1].

The effect that hinders the spreading of the liquid over the plate is therefore the surface tension, since it tends to carry the liquid towards the central part of the domain instead of extending it to increase the gas-liquid surface area. One can avoid the effect of surface tension by using geometric patterns on the solid surface in order to channel the flow conveniently. This would avoid the exchange of momentum between both the parallel and the transversal direction to the flow, which hinders the liquid spreading. The use of a geometric pattern on the solid surface was reported by Sebastia-Saez et al. [21] as having the effect of increasing the gas-liquid interface area. Further work will consist in studying the effect of those patterns on the formation of braids and meanders.

\section{Conclusions}

A CFD approach was used to study the braiding and meandering phenomena on rivulets flowing down inclined channels. Mertens et al. [1] reported a correlation to predict whether braids form or not depending on the physical properties of the fluid, the flow rate and the inclination of the plate. Five cases with various values of the liquid flow rate were tested and classified satisfactorily according to the correlation.

The oscillations of the rivulet upon meandering conditions are quantified. With an inlet velocity $v_{\text {inlet }}=25 \mathrm{~cm} / \mathrm{s}$ and $\alpha=60^{\circ}$, the oscillations show a frequency of $5.6 \mathrm{~Hz}$, with an amplitude of $1.5 \mathrm{~mm}$. CFD allows to consider a perfectly smooth surface, which leads to the conclusion that the oscillations respond to imbalances in the terms of the Navier-Stokes equations that govern 
the flow as suggested by Daerr et al. [14]. Further work will include the analysis of the terms in the Navier-Stokes equations in order to deepen in the roots of this behaviour as well as longer computational domains to allow comparing between different meandering cases.

As for braiding, the velocity isolines within the liquid film are analysed, confirming the theory presented by Mertens et al. [1] by which the cause of the braiding formation is the readjustment between the inertia of the liquid and the surface tension. Acceleration of the liquid in the area with a full film flow is observed, followed by the formation of two retraction waves, which bring the liquid back to the centre line. CFD combined with the Volume of Fluid (VOF) algorithm allows to analyse the velocity field within the rivulet flow. The effect of the surface tension becomes evident by analysing the $x$-dir component of the velocity at those waves, which increases as the liquid flows downwards to the braid. In order to reduce the braiding instability, it would therefore be interesting to reduce the increase of momentum in the transversal direction, and thus, avoiding the effect of the surface tension.

In order to increase the gas-liquid interface available for mass transfer, one should therefore limit the effect of the surface tension. Using solid surfaces with geometric patterns can help to achieve it by channelling the flow and reducing the formation of braids and meanders. Further work will consist in designing new geometric patterns to avoid the effect of the surface tension.

Overall, this work represents a new perspective to understand the complex processes of braiding and meandering in solid surfaces, which are strongly relevant phenomena in a number of chemical engineering applications such as separation units, heat exchangers, and the emerging technology of microchannel reactors. Our work shed also some light on the theoretical understanding of these processes while opening new research avenues for experimental work. 


\section{Acknowledgements}

The authors gratefully acknowledge the financial support from the EPSRC (United Kingdom): Project grant EP/J020184/1; and FP7 Marie Curie iComFluid project grant 312261. 


\section{Symbols used}

F

$F_{S}$

$f$

GCI

$g$

$h$

$N$

$p$

$\tilde{p}$

$q$

Re

$r$

$S_{m}$

$S_{\mu}$

$S_{\sigma}$

$t$

$v$

$x, y, z$

\section{Greek symbols}

$\alpha$

$\gamma$

$\delta$
$[-]$

[-]

$\left[\mathrm{s}^{-1}\right]$

$[-]$

$\left[\mathrm{m} \mathrm{s}^{-2}\right]$

[m]

$[-]$

$\left[\mathrm{kg} \mathrm{s}^{-2} \mathrm{~m}^{-1}\right]$

$[-]$

$\left[\mathrm{m}^{3} \mathrm{~s}^{-1}\right]$

$[-]$

$[-]$

$\left[\mathrm{kg} \mathrm{m}^{-3} \mathrm{~s}^{-1}\right]$

$\left[\mathrm{kg} \mathrm{m}^{-2} \mathrm{~s}^{-2}\right]$

$\left[\mathrm{kg} \mathrm{m}^{-2} \mathrm{~s}^{-2}\right]$

[s]

$\left[\mathrm{m} \mathrm{s}^{-1}\right]$

$[-]$

$\left[\mathrm{kg} \mathrm{s}^{-2}\right]$

[m] volume fraction

safety factor

frequency of oscillation

grid convergence index

acceleration of gravity

grid spacing

number of nodes in a particular grid

pressure

apparent convergence rate

volumetric flow rate

Reynolds number

grid refinement ratio

mass source term

momentum source term due to variations in dynamic

viscosity

momentum source term due to surface tension

time

velocity

denote coordinate directions

inclination of the channel over the horizontal plane

surface tension coefficient

velocity inlet thickness 
$\kappa$

$\mu$

$v$

$\Pi_{1}, \Pi_{2}$

$\rho$

$\tau$

$\phi$

\section{Subscripts}

\section{$1,2,3$}

exact

$g$

inl

1

norm

\section{Abbreviations}

CFD

CSF

HOT

VOF $\left[\mathrm{m}^{-1}\right]$

$\left[\mathrm{kg} \mathrm{s}^{-1} \mathrm{~m}^{-1}\right]$

$\left[\mathrm{m}^{2} \mathrm{~s}^{-1}\right]$

$[-]$

$\left[\mathrm{kg} \mathrm{m}^{-3}\right]$

$[-]$

normalised time

generic variable

relative error

curvature

density solid-liquid contact angle

dynamic viscosity

kinematic viscosity

parameters in Mertens et al. correlation [1]

Denote fine, medium and coarse mesh, respectively

exact solution

gas phase

inlet conditions

liquid Phase

normalised

Computational Fluid Dynamics

higher order terms

Volume of Fluid 


\section{References}

[1] K. Mertens, V. Putkaradze, P. Vorobieff, Nature. 2004, 430, 165. DOI:10.1038/430165a

[2] T. Nakagawa, Int. J. Multiphase Flow. 1992, 18 (3), 455. DOI:

https://doi.org/10.1016/0301-9322(92)90028-F

[3] P. Schmuki, M. Laso. J. Fluid Mech. 1990, 215, 125. DOI:

https://doi.org/10.1017/S0022112090002580

[4] T. Nakagawa, J.C. Scott. J. Fluid Mech. 1984, 149, 89. DOI:

https://doi.org/10.1017/S002211208400255X

[5] M.A. Herrada, A.S. Mohamed, J.M. Montanero, A.M. Gañán-Calvo, Int. J. Multiphase Flow. 2015, 69, 1. DOI: https://doi.org/10.1016/j.ijmultiphaseflow.2014.10.012

[6] B. De, T.K. Mandal, G. Das, Therm Fluid Sci. 2010, 34 (5), 625. DOI:

https://doi.org/10.1016/j.expthermflusci.2009.12.002

[7] R.K. Singh, J.E. Galvin, X. Sun, Chem. Eng. Sci. 2016, 142, 244. DOI:

https://doi.org/10.1016/j.expthermflusci.2009.12.002

[8] Y.Y. Xu, S. Paschke, J.-U. Repke, J.Q. Yuan, G. Wozny, Chem. Eng. Technol. 2009, 32, 1227. DOI: 10.1002/ceat.200900099

[9] L. Raynal, A. Royon-Lebeaud, Chem. Eng. Sci. 2007, 62 (24), 7196. DOI: https://doi.org/10.1016/j.ces.2007.08.010

[10] A. Hoffmann, I. Ausner, J.-U. Repke, G. Wozny. Comput. Chem. Eng. 2005, 29 (6), 1433. DOI: https://doi.org/10.1016/j.compchemeng.2005.02.004

[11] K. Mertens, V. Putkaradze, P. Vorobieff, J. Fluid Mech. 2005, 531, 49. DOI:

https://doi.org/10.1017/S0022112005003873 
[12] B. Birnir, K. Mertens, V. Putkaradze, P. Vorobieff, J. Fluid Mech. 2008, 607, 401. DOI: https://doi.org/10.1017/S0022112008002000

[13] Y. Iso, X. Chen, J. Fluids Eng. 2011, 133 (9), 091101. DOI:10.1115/1.4004765

[14] A. Daerr, J. Eggers, L. Limat, N. Valade, Phys. Rev. Lett. 2011, 106 (18), 184501. DOI: https://doi.org/10.1103/PhysRevLett.106.184501

[15] H.Y. Kim, J.H. Kim, B.H. Kang, J. Fluid Mech. 2004, 498, 245. DOI:

https://doi.org/10.1017/S0022112003006748

[16] Y. Yoshimura, Y. Yagisawa, K. Okumura, Nat. Scientific Reports. 2016, 6, 38457. DOI: $10.1038 /$ srep38457

[17] Y. Haroun, L. Raynal, D. Legendre, Chem. Eng. Sci. 2012, 75, 342. DOI: https://doi.org/10.1016/j.ces.2012.03.011

[18] J. Brackbill, D. Kothe, C. Zemach, J. Comput. Phys. 1992, 100 (2), 335. DOI: https://doi.org/10.1016/0021-9991(92)90240-Y

[19] P.J. Roache, Verification and validation in computational science and engineering, 1st ed., Hermosa Publishers, Albuquerque (NM) 1998

[20] M.S.M. Ali, C.J. Doolan, V. Wheatley, presented at 7th Int. Conf. on CFD in the Minerals and Process Industries (CSIRO), Melbourne, Australia, December 2009 [21] D. Sebastia-Saez, S. Gu, P. Ranganathan, K. Papadikis, Int J. Greenhouse Gas Control. 2013, 19, 492. DOI: https://doi.org/10.1016/j.ijggc.2013.10.013 
Tables with headings

Table 1 Details of the computational grids. From fine to coarse, the grids are identified with the numbers 1 to 3. These identifiers will be used as subscripts in Eqns. (5) to (9).

\begin{tabular}{|c|c|c|c|c|c|}
\hline Grid Level & Nodes $x$-dir & Nodes y-dir & Nodes Z-dir & $\begin{array}{l}\text { Total nodes } \\
(N)\end{array}$ & $\begin{array}{l}\text { Grid spacing }(h) \\
{[\mu \mathrm{m}]}\end{array}$ \\
\hline \multicolumn{6}{|c|}{ Case $1\left(v_{i n l}=25 \mathrm{~cm} / \mathrm{s} ; \alpha=45^{\circ}\right)$} \\
\hline Coarse (3) & 28 & 53 & 117 & 173,628 & 10.5 \\
\hline Medium (2) & 31 & 59 & 132 & 241,428 & 9.5 \\
\hline Fine (1) & 35 & 67 & 147 & 344,715 & 8.3 \\
\hline \multicolumn{6}{|c|}{ Case $3\left(v_{\text {inl }}=25 \mathrm{~cm} / \mathrm{s} ; \alpha=60^{\circ}\right)$} \\
\hline Coarse (3) & 42 & 78 & 177 & 579,852 & 13,8 \\
\hline Medium (2) & 50 & 93 & 212 & 985,800 & 11.4 \\
\hline Fine (1) & 60 & 112 & 254 & 1706,880 & 9.3 \\
\hline
\end{tabular}


Table 2 Summary of the conditions of the six cases tested. The thickness $\delta$ of the liquid inlet is indicated in bold.

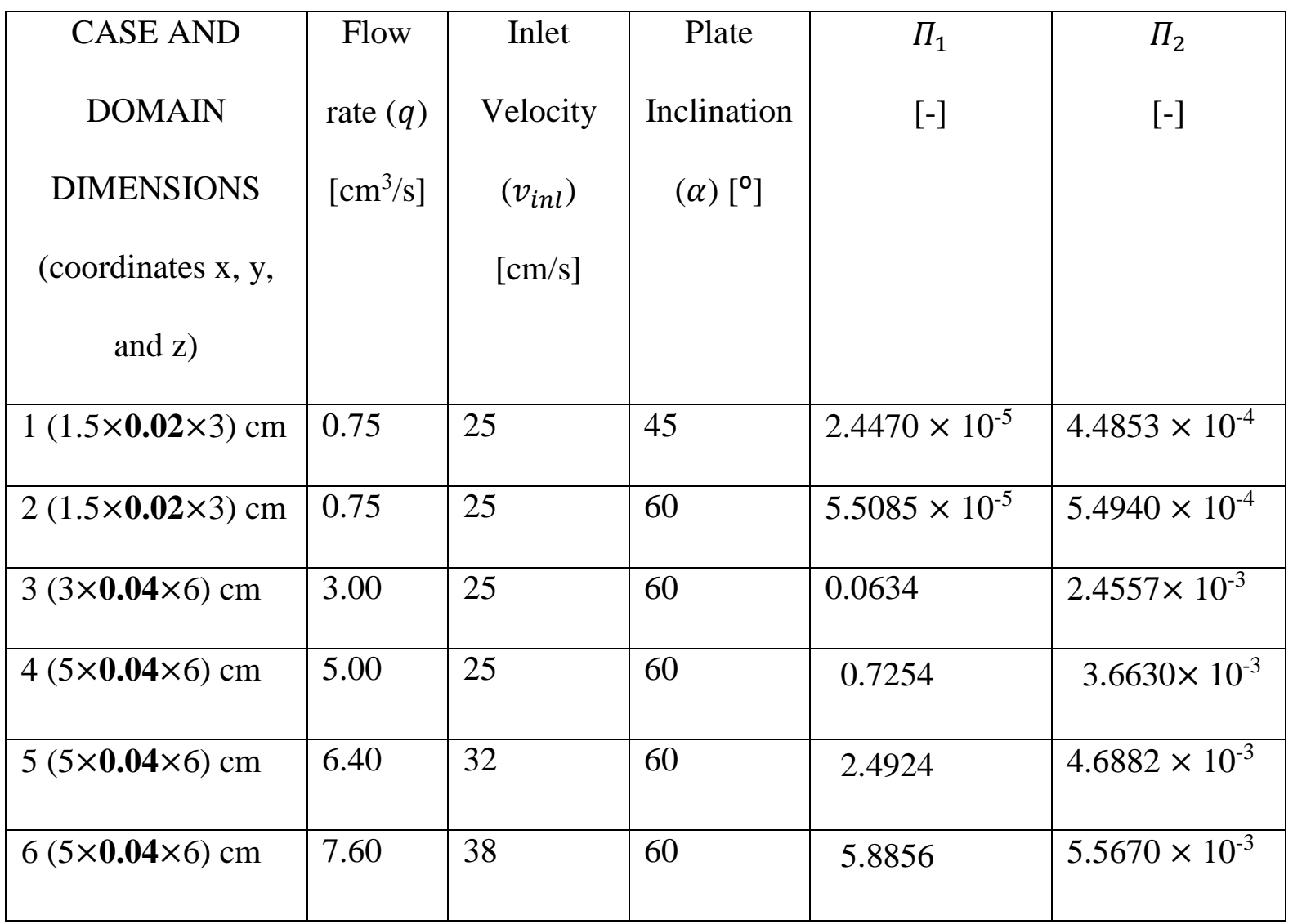

\section{Short text for the table of contents section}

Rivulet instabilities (meandering and braiding) affect the interface area available for mass transfer in separation equipment, reducing the efficiency. CFD shows that, at low flow rates, oscillations of the rivulet occur. At greater flow rates, the effect of the surface tension, which causes the braiding, on the transversal momentum, is highlighted. 